\title{
From fuzzy sets to interval-valued and Atanassov intuitionistic fuzzy sets: a unified view of different axiomatic measures
}

\author{
Inés Couso, and Humberto Bustince, Senior member, IEEE
}

\begin{abstract}
We examine a broad collection of axiomatic definitions from various and diverse contexts, within the domain of fuzzy sets. We evaluate their respective extensions to the case of interval-valued fuzzy sets and intuitionistic fuzzy sets, from a purely formal point of view. We conclude that a large number of such extensions follow similar formal procedures This fact allows us to formulate a general procedure which encompasses all the reviewed extensions as particular cases of it. The new general formulation allows us to identify three different procedures to derive the corresponding extension to the field of interval-valued fuzzy sets or to the field of intuitionistic fuzzy sets from a specific real-valued measure in the context of fuzzy sets. These three processes agglutinate a multitude of particular constructions found in the literature.
\end{abstract}

Index Terms-Interval-valued fuzzy sets, Atanassov intuitionistic fuzzy sets, extensions of fuzzy sets

\section{INTRODUCTION}

The notion of interval-valued fuzzy set (IVF set) was proposed in 1975 independently by Zadeh [45], Grattan-Guiness [20] and Sambuc [35], as a generalization of the notion of fuzzy set. IVF sets are defined by means of interval-valued membership functions. About 10 years later, Atanassov [1], [2] introduced the notion of intuitionistic fuzzy set (IF set), characterized by a pair of functions, the first one determining the "degree of membership" and the second one reflecting the "degree of non-membership". The sum of both degrees should be never greater than 1. Despite the semantic differences, intuitionistic fuzzy sets where proved to be formally equivalent to interval-valued fuzzy sets by several authors, including Atanassov ( [3], [16]).

Since the introduction of both notions (see [9] for a detailed historical account), many authors have adapted / generalized different definitions that had been originally proposed for fuzzy sets (FS) to these more general contexts. Many of those original notions are characterized in terms of real-valued functions defined on a family of tuples of fuzzy sets. This is the case for instance, of the notion of the probability of fuzzy events, or the degrees of inclusion, similarity, distance, resemblance, or divergence between two fuzzy sets, among many others. We can separate those generalizations into two main groups, depending on whether the generalized mapping is defined as a set-valued function or a real-valued mapping.

I. Couso is with the Department of Statistics and O.R., University of Oviedo, Spain. E-mail: couso@uniovi.es.

Humberto Bustince is with Departamento de Automática y Computación and Institute of Smart Cities, Universidad Pública de Navarra, Spain. E-mail: bustince@unavarra.es.
We can distinguish in turn two different kinds of generalizations that we will respectively refer to as "constructive" and "axiomatic".

In the first case (constructive definitions), specific (families of) formulas extending existing FS formulations to these more general contexts are proposed. The different generalizations lead either to interval-valued or numerical-valued measures. The notion of "probability" of intuitionistic fuzzy events initially proposed by Szmidt and Kacprzyk [38] and later on considered by Grzegorzewski [21], the "degree of compatibility" between IVF sets of Gorzalczany [19], the "degree of inclusion" studied by Bustince [6] and Grzegorzewski [23], the notion of "cardinality" independently proposed by several authors [34], [40], [42]) in order to extend the notion of "sigma-count" or "cardinality" of a fuzzy set [30] are examples of constructive set-valued extensions. Those setvalued constructions have been extensively analyzed in [11]. Alternatively, other authors have proposed some specific scalar constructions for different notions. This is the case of many definitions of similarity between IF sets (some examples can be found in [15], [24], [26], [27], [44]), distance (see [22], [25], [39], [41] for instance) among many others.

In the second case, axiomatic definitions that somehow extend or adapt the original definition from the fuzzy set context to this more general context are given. There are several set-valued extensions of this kind. To give some examples, a collection of axioms regarding the notion of "setvalued inclusion" and extending the initial axioms proposed by Sinha-Dougherty [36] in the particular context of fuzzy sets was proposed by Cornelis and Kerre in [10]. Similarly, lists of axioms regarding the notion of set-valued similarity have been independently proposed by Galar et al. in [18], and Stachowiak-Dyczkowski in [37]. We can also find some examples of axiomatic scalar extensions. This is the case of the notion of similarity between two IF sets (two different variants can be found in [15] and [48]), distance [48] or divergence [32].

In our paper, we will analyze this second stream of the literature from a formal perspective. We will show that many different axiomatic extensions follow exactly the same formal pattern even if they deal with completely different notions. This will allow us to simplify the proofs of many results studying implication relations between different variants of the same extended notion, based on previous studies about the corresponding original notions in the context of fuzzy sets. It will also provide us with standarized methods of construction 
of extended measures, that will encompass as particular cases many specific constructions from the recent literature. We will divide our analysis into two parts: the first one will deal with set-valued generalizations and the second-one will deal with real-valued generalizations. Both types of generalizations will be respectively studied in Sections III and IV.

\section{PRELIMINARIES}

Let $U$ denote the universe of discourse and let $\wp(U)$ be its power set. Let $\mathcal{F}(U)$ denote the family of fuzzy subsets of $U$. A fuzzy subset of $U, A \in \mathcal{F}(U)$ is a mapping $A: U \rightarrow$ $[0,1]$. For each element $x \in U$, the value $A(x)$ is called the "membership degree" of $x$ to the fuzzy set $A$. An Atanassov intuitionistic fuzzy set is a mapping $A: U \rightarrow D([0,1])=$ $\{(x, y) \in[0,1]: x+y \leq 1\}$. It is therefore represented by means of a pair of mappings $\mu_{A}: U \rightarrow[0,1]$ and $\nu_{A}: U \rightarrow$ $[0,1]$ respectively called the "degree of membership" and of "non-membership" satisfying the restriction

$$
\mu_{A}(x)+\nu_{A}(x) \leq 1, \forall x \in U .
$$

The difference $\pi_{A}(x)=1-\nu_{A}(x)-\mu_{A}(x)$ is seen as a hesitation degree of the expert in order to assign a numerical membership value to $x$. Therefore, intuitionistic fuzzy sets are seen as generalizations of fuzzy sets, in the sense that the last ones are IF sets with null hesitation degree. In fact, $\pi_{A}(x)=$ $0, \forall x \in U$, if and only if $\mu_{A}(x)=1-\nu_{A}(x), \forall x \in U$, and therefore any IF set will null hesitation degree is univocally characterized by the membership function $\mu_{A}: U \rightarrow[0,1]$.

An interval-valued fuzzy subset of $U$ is an interval-valued mapping $A=[\underline{A}, \bar{A}]: U \rightarrow \mathbb{I}([0,1])$, where $\mathbb{I}([0,1])$ denotes the family of closed intervals included in the unit interval $[0,1]$. It is therefore characterized by the mappings $\underline{A}: U \rightarrow$ $[0,1]$ and $\bar{A}: U \rightarrow[0,1]$, with $\underline{A}(x) \leq \bar{A}(x), \forall x \in U$, respectively determining the lower and upper bounds of the corresponding intervals. If $\underline{A}(x)=\bar{A}(x), \forall x \in U$, then $A$ is considered to be a fuzzy set.

We can define a bijection, $\Phi: I V F S(U) \rightarrow I F S(U)$, between the collection of IVF sets and the collection of IF sets, such that any interval-valued fuzzy set $A=[\underline{A}, \bar{A}]$ is mapped to the IF set $A^{\prime}=\left(\mu_{A}, \nu_{A}\right)$ where $\mu_{A}$ and $\nu_{A}$ are respectively defined as:

$$
\mu_{A}(x)=\underline{A}(x), \text { and } \nu_{A}(x)=1-\bar{A}(x), \forall x \in U .
$$

Therefore, any mapping defined over a class of $k$-tuples of IF sets can be straightforwardly identified with a mapping defined over $k$-tuples of IVF sets.

Along the paper, we will consider the following notion of inclusion between IVF sets (or formally equivalently, between the corresponding IF sets [2]): An interval-valued fuzzy set $A=[\underline{A}, \bar{A}] \in I V F S(U)$ is said to be included in $B=$ $[\underline{B}, \bar{B}] \in I V F S(U)$ if and only if $\underline{A} \subseteq \underline{B}$ and $\bar{A} \subseteq \bar{B}$, where $\subseteq$ denotes the usual inclusion between fuzzy sets. We will denote it as $A \subseteq_{L} B$. We will also use the so-called lattice ordering between closed intervals of the real line: an interval $[\underline{a}, \bar{a}]$ will be said to be less than or equal to another interval $[\underline{b}, \bar{b}]$ if and only if $\underline{a} \leq \underline{b}$ and $\bar{a} \leq \bar{b}$. We will denote it as $[\underline{a}, \bar{a}] \leq_{L}[\underline{b}, \bar{b}]$.
We will consider the usual operations of intersection, union and complement as follows: consider two IVF sets $A=[\underline{A}, \bar{A}]$ and $B=[\underline{B}, \bar{B}]$.

- Their intersection is the IVF set $A \cap B=[\underline{A \cap B}, \overline{A \cap B}]$ with $\underline{A \cap B}(x)=(\underline{A} \cap \underline{B})(x)=\min \{\underline{A}(x), \underline{B}(x)\}$, and $\overline{A \cap B}(x)=(\bar{A} \cap \bar{B})(x)=\min \{\bar{A}(x), \bar{B}(x)\}$

- Their union is the IVF set $A \cup B=[\underline{A \cup B}, \overline{A \cup B}]$ with $\underline{A \cup B}(x)=(\underline{A} \cup \underline{B})(x)=\max \{\underline{A}(x), \underline{B}(x)\}$, and $\overline{A \cup B}(x)=(\bar{A} \cup \bar{B})(x)=\max \{\bar{A}(x), \bar{B}(x)\}$.

- Consider the usual complement on $\mathcal{F}(U)$ defined as $X^{c}(x)=1-X(x), \forall x \in U$ and all $X \in \mathcal{F}(U)$. The complement of $A$ is $A^{c}=\left[\underline{A^{c}}, \overline{A^{c}}\right]$ with $\underline{A^{c}}=\bar{A}^{c}$ and $\overline{A^{c}}=\underline{A}^{c}$.

We will study different extensions of real-valued mappings defined over sets of $k$-tuples of fuzzy sets i.e., extensions of functions of the form $f: \mathcal{F}(U) \times(k) \times \mathcal{F}(U) \rightarrow \mathbb{R}$. The collection of $k$-tuples of fuzzy sets will be shortly denoted by $\mathcal{F}^{k}(U)$, while the collection of $k$-tuples of IVF sets will be denoted by $I V F^{k}(U)$. Given an element $\boldsymbol{A}=\left(A_{1}, \ldots, A_{k}\right)$ of $I V F^{k}(U)$, with $A_{i} \in\left[A_{i}, \overline{A_{i}}\right] \in I V F S(U), i=1, \ldots, k$, $\underline{\boldsymbol{A}} \in \mathcal{F}^{k}(U)$ and $\overline{\boldsymbol{A}} \in \overline{\mathcal{F}^{k}}(U)$ will respectively denote the tuples $\underline{\mathbf{A}}=\left(A_{1}, \ldots, A_{k}\right)$ and $\overline{\mathbf{A}}=\left(\overline{A_{1}}, \ldots, \overline{A_{k}}\right)$. Given $X=\left(X_{1}, \ldots, X_{k}\right) \bar{\epsilon} F^{k}(U)$, and $x \in U, X(u)$ will denote the following $k$-tuple of membership values $X(x)=$ $\left(X_{1}(x), \ldots, X_{k}(x)\right)$.

\section{SET-VALUED GENERALIZATIONS}

In this section we will deal with set-valued extensions of concepts originally defined within the context of fuzzy sets. In [11], we have reviewed three different but related construction methods of set-valued generalizations, the socalled set-valued, max-min and max-min-varied extensions. Each of those construction methods includes, as particular cases, several particular definitions from the literature. Although less common, we can also find some works in the literature proposing lists of axioms that certain set-valued measures should satisfy. This is the case of the notion of set-valued inclusion measures of Cornelis-Kerre [10], and the three variants of the notion of interval-valued similarity measures respectively introduced by Bustince [6] Galar et al. [18] and Stachowiak-Dyczkowski [37]. Let us recall Galar et al. definition [18] as an illustration of the general definition to be provided in this section (Definition 6):

Definition 1: [18] $s: \operatorname{IVF} S^{2}(U) \rightarrow \mathbb{I}([0,1])$ is an IV fuzzy similarity measure if:

- $s(A, B)=s(B, A), \forall(A, B) \in I V F S^{2}(U)$ (Symmetry)

- $s\left(A, A^{c}\right)=\{0\}$ iff $A$ is a crisp set. (Minimum value)

- $s(A, B)=\{1\}$ iff $A=B$. (Maximum value)

- If $A \subseteq \subseteq_{L} B \subseteq \subseteq_{L} \quad C$ then $s(A, C) \leq_{L} \quad s(A, B)$ and $s(A, C) \leq_{L} s(B, C)$. (Monotonicity wrt inclusion)

- $s\left(A^{c}, B^{c}\right)=s(A, B), \forall(A, B) \in I V F S(U)$. (Complement)

The above definition shares some commonalities with a previous notion of similarity introduced by Bustince [6] and with the notion of set-valued inclusion measure introduced by Cornelis and Kerre [10], even if this last one deals with 
a completely different concept. All of them can be seen as instances of the general notion established in Definition 6.

We first need to provide some auxiliary definitions.

Definition 2: Let us consider an arbitrary subset of $\mathbb{R}^{n}, C \subseteq$ $\mathbb{R}^{n}$. We define the rectangle projection of $C$ as the collection $T(C)$ of $n$-dimensional rectangles defined as follows:

$$
\begin{array}{r}
T(C)=\left\{\left[a_{1}, b_{1}\right] \times \ldots \times\left[a_{n}, b_{n}\right]: \vec{a}=\left(a_{1}, \ldots, a_{n}\right)\right. \text { and } \\
\left.\vec{b}=\left(b_{1}, \ldots, b_{n}\right) \in C\right\} .
\end{array}
$$

The next definition refers to two types of general properties. Particular instances of them are involved in many axiomatic definitions of similarities, dissimilarities, divergences, etc, in the context of fuzzy sets.

Definition 3: Let us consider a universe $U$ and some $k \in \mathbb{N}$. Let us consider an arbitrary mapping $f: \mathcal{F}^{k}(U) \rightarrow \mathbb{R}$. Let us consider two sets of tuples $\mathcal{D}, \mathcal{F} \subseteq \mathcal{F}^{k}(U) \times(n) \times \mathcal{F}^{k}(U)$ and $C \subseteq \mathbb{R}^{n}$.

- $f$ is said to satisfy property $\mathbf{P}=P^{1}(\mathcal{D}, \mathcal{F}, C)$ if, given $\left(\boldsymbol{A}_{1}, \ldots, \boldsymbol{A}_{n}\right) \in \mathcal{D}$,

$$
\left(\boldsymbol{A}_{1}, \ldots, \boldsymbol{A}_{n}\right) \in \mathcal{F} \Rightarrow\left(f\left(\boldsymbol{A}_{1}\right), \ldots, f\left(\boldsymbol{A}_{n}\right)\right) \in C .
$$

$\mathbf{P}$ is said to be a property of type 1 .

- $f$ is said to satisfy property $\mathbf{P}^{\prime}=P^{2}(\mathcal{D}, \mathcal{F}, C)$ if, given $\left(\boldsymbol{A}_{1}, \ldots, \boldsymbol{A}_{n}\right) \in \mathcal{D}$

$$
\left(\boldsymbol{A}_{1}, \ldots, \boldsymbol{A}_{n}\right) \in \mathcal{F} \Leftrightarrow\left(f\left(\boldsymbol{A}_{1}\right), \ldots, f\left(\boldsymbol{A}_{n}\right)\right) \in C .
$$

$\mathbf{P}^{\prime}$ is said to be a property of type 2 .

Remark 1: When $\mathcal{D}$ represents the whole family of tuples $\mathcal{F}^{k}(U) \times \stackrel{(n)}{.} \times \mathcal{F}^{k}(U)$, then we will use the simpler notation $\mathbf{P}=P^{1}(\mathcal{F}, C)$ and $\mathbf{P}^{\prime}=P^{2}(F, C)$ instead of $\mathbf{P}=P^{1}(\mathcal{D}, \mathcal{F}, C)$ and $\mathbf{P}^{\prime}=P^{2}(\mathcal{D}, \mathcal{F}, C)$.

Definition 4: Consider a sequence $\boldsymbol{P}_{1}, \ldots, \boldsymbol{P}_{l}$, where $\boldsymbol{P}_{i}$ is either a property of type 1 or type $2, i=1, \ldots, l$. A mapping $f: \mathcal{F}^{k}(U) \rightarrow \mathbb{R}$ is said to satisfy the notion

$$
\boldsymbol{N}=N\left(\boldsymbol{P}_{1}, \ldots, \boldsymbol{P}_{l}\right)
$$

if it satisfies all the above $l$ properties.

Example 1: Let us consider the following definition of "similarity" between fuzzy sets [15]. $s: \mathcal{F}^{2}(U) \rightarrow \mathbb{R}$ is a similarity between fuzzy sets if it satisfies:

- Sim 1) $0 \leq s(A, B) \leq 1, \forall(A, B) \in \mathcal{F}^{2}(U)$.

- Sim 2) $s(A, B)=1 \Leftrightarrow A=B$.

- Sim 3) $s(A, B)=s(B, A), \forall(A, B) \in \mathcal{F}^{2}(U)$.

- Sim 4) If $A \subseteq B \subseteq C$ then $s(A, C) \leq s(A, B)$.

- Sim 5) If $A \subseteq B \subseteq C$ then $s(A, C) \leq s(B, C)$.

Properties Sim 1, Sim 3, Sim 4 and Sim 5 can be regarded as type 1 properties (characterized by one side implications), while Property Sim 2 is a type 2 property (based on an equivalence relation). In fact, we can alternatively formulate the five of them as follows:

- Sim 1) $s$ satisfies $P^{1}\left(\mathcal{F}_{1}, C_{1}\right)$ with $\mathcal{F}_{1}=\mathcal{F}^{2}(U)$ and $C_{1}=[0,1]$.

- Sim 2) $s$ satisfies $P^{2}\left(\mathcal{F}_{2}, C_{2}\right)$ with $\mathcal{F}_{2}=\{(X, X): X \in$ $\mathcal{F}(U)\}$ and $C_{2}=\{1\}$.

- $\operatorname{Sim} 3) s$ satisfies $P^{1}\left(\mathcal{F}_{3}, C_{3}\right)$, where $\mathcal{F}_{3}=$ $\left\{(X, Y),(Z, T) \in \mathcal{F}^{2}(U) \times \mathcal{F}^{2}(U): X=T, Y=Z\right\}$ and $C_{3}=\{(x, x): x \in[0,1]\}$.
- $\operatorname{Sim} 4) s$ satisfies $P^{1}\left(\mathcal{F}_{4}, C_{4}\right)$ with $\mathcal{F}_{4}=$ $\left\{(X, Y),(Z, T) \in \mathcal{F}^{2}(U) \times \mathcal{F}^{2}(U): X=Z, Y \subseteq Z\right\}$ and $C_{4}=\left\{(x, y) \in \mathbb{R}^{2}: x \geq y\right\}$.

- $\operatorname{Sim} 5) s$ satisfies $P^{1}\left(\mathcal{F}_{5}, C_{4}\right)$ with $\mathcal{F}_{5}=$ $\left\{(X, Y),(Z, T) \in \mathcal{F}^{2}(U) \times \mathcal{F}(U) \times \mathcal{F}^{2}(U): X \subseteq\right.$ $Z, Y=Z\}$.

Definition 5: Let us consider two sets of tuples $\mathcal{D}, \mathcal{F} \subseteq$ $\mathcal{F}^{k}(U) \times \stackrel{(n)}{.} \times \mathcal{F}^{k}(U)$ and $C \subseteq \mathbb{R}^{n}$ for some $n \in \mathbb{N}$. Let us also consider two mappings $G^{1}, G^{2}: I V F S^{k}(U) \rightarrow \mathcal{F}^{k}(U)$.

We say that $f=[f, \bar{f}]: I V F S^{k}(U) \rightarrow \mathbb{R}$ satisfies the $\left(G^{1}, G^{2}\right)$-interval-extension of $\mathbf{P}=P^{1}(\mathcal{D}, \mathcal{F}, C), I_{G^{1}, G^{2}}(\mathbf{P})$ if:

$$
\begin{gathered}
\left(G^{1}\left(\boldsymbol{A}_{1}\right), \ldots, G^{1}\left(\boldsymbol{A}_{n}\right)\right) \in \mathcal{F} \text { and } \\
\left(G^{2}\left(\boldsymbol{A}_{1}\right), \ldots, G^{2}\left(\boldsymbol{A}_{n}\right)\right) \in \mathcal{F} \Rightarrow \\
\left(\underline{f}\left(\boldsymbol{A}_{1}\right), \ldots, \underline{f}\left(\boldsymbol{A}_{n}\right)\right) \in C \text { and }\left(\bar{f}\left(\boldsymbol{A}_{1}\right), \ldots, \bar{f}\left(\boldsymbol{A}_{n}\right)\right) \in C .
\end{gathered}
$$

Analogously, we say that $f=[f, \bar{f}]: I V F S^{k}(U) \rightarrow$ $\mathbb{I}$ satisfies the $\left(G^{1}, G^{2}\right)$-interval-extension of $\mathbf{P}^{\prime}=$ $P^{2}(\mathcal{D}, \mathcal{F}, C), \quad I_{\left(G^{1}, G^{2}\right)}\left(\boldsymbol{P}^{\prime}\right)$ if, given $\left(\boldsymbol{A}_{1}, \ldots, \boldsymbol{A}_{n}\right) \in$ $I V F S^{k}(U) \times \stackrel{(n)}{.} I V F S^{k}(U)$ with $\left(G^{1}\left(\boldsymbol{A}_{1}\right), \ldots, G^{1}\left(\boldsymbol{A}_{n}\right)\right) \in$ $\mathcal{D}$ and $\left(G^{2}\left(\boldsymbol{A}_{1}\right), \ldots, G^{2}\left(\boldsymbol{A}_{n}\right)\right) \in \mathcal{D}$, the following equivalence holds:

$$
\begin{gathered}
\left(G^{1}\left(\boldsymbol{A}_{1}\right), \ldots, G^{1}\left(\boldsymbol{A}_{n}\right)\right) \in \mathcal{F}, \text { and } \\
\left(G^{2}\left(\boldsymbol{A}_{1}\right), \ldots, G^{2}\left(\boldsymbol{A}_{n}\right)\right) \in \mathcal{F}
\end{gathered}
$$

if and only if

$$
\left(\underline{f}\left(\boldsymbol{A}_{1}\right), \ldots, \underline{f}\left(\boldsymbol{A}_{n}\right)\right) \in C \text { and }\left(\bar{f}\left(\boldsymbol{A}_{1}\right), \ldots, \bar{f}\left(\boldsymbol{A}_{n}\right)\right) \in C .
$$

Remark 2: When, in particular $G^{1}(\boldsymbol{A})=\underline{A}$ and $G^{2}(\boldsymbol{A})=$ $\bar{A}$, we just say that $f=[\underline{f}, \bar{f}]$ is the (canonical) intervalextension of $f$.

Definition 6: Consider a sequence $\boldsymbol{P}_{1}, \ldots, \boldsymbol{P}_{l}$, where $\boldsymbol{P}_{i}$ is either a property of type 1 or type $2, i=1, \ldots, l$. Consider the notion $\boldsymbol{N}=N\left(\boldsymbol{P}_{1}, \ldots, \boldsymbol{P}_{l}\right)$

We say that $I: I V F S^{k}(U) \rightarrow \mathbb{R}$ satisfies the $\left(\left(G_{1}^{1}, G_{1}^{2}\right), \ldots,\left(G_{l}^{1}, G_{l}^{2}\right)\right)$-interval-extension of $\mathbf{N}$, $I_{\left(G_{1}^{1}, G_{1}^{2}\right), \ldots,\left(G_{l}^{1}, G_{l}^{2}\right)}(\boldsymbol{N})$ if it satisfies the $\left(G_{i}^{1}, G_{i}^{2}\right)$-interval extension of $\boldsymbol{P}_{i}$ for every $i=1, \ldots, l$.

Example 2: Let us check that the notion of interval-valued similarity measure considered in Definition 1 is a particular instance of Definition 6. In fact, its characterizing properties can be alternatively expressed as follows. A mapping $s: I V F S^{2}(U) \rightarrow \mathbb{R}$ is an interval-valued similarity measure if it satisfies:

- The (canonical) interval-extension of $P^{1}\left(\mathcal{F}_{1}, C_{1}\right)$, $I\left(P^{1}\left(\mathcal{F}_{1}, C_{1}\right)\right)$, where $\mathcal{F}_{1}=\{((X, Y),(Z, T)) \in$ $\left.\mathcal{F}^{2}(U) \times \mathcal{F}^{2}(U): X=T, Y=Z\right\}$ and $C_{1}=\{(x, x): x \in \mathbb{R}\}$. (Symmetry)

- The $\left(G^{1}, G^{2}\right)$ - interval extension $I_{G_{1}^{1}, G_{1}^{2}}\left(P^{2}\left(\mathcal{D}, \mathcal{F}_{2}, C_{2}\right)\right)$ where $G_{1}^{1}(A, B)=(\underline{A}, \bar{B})$, $G_{1}^{2}(A, B)=(\bar{A}, \underline{B}), \mathcal{D}=\left\{(A, B) \in \mathcal{F}^{2}(U): A^{c}=\right.$ $B\}, \mathcal{F}_{2}=\left\{(A, B) \in \mathcal{F}^{2}(U): A, B \in \wp(U)\right\}$, and $C_{2}=\{0\}$. (Minimum value)

- The interval-extension $I\left(P^{2}\left(\mathcal{F}_{3}, C_{3}\right)\right)$, where $\mathcal{F}_{3}=$ $\left\{(A, B) \in \mathcal{F}^{2}(U): A=B\right\}$, and $C_{3}=\{1\}$. (Maximum value) 
- The interval-extension $I\left(P^{1}\left(\mathcal{F}_{4}, C_{4}\right)\right)$, where $\mathcal{F}_{4}=$ $\left\{((A, B),(C, D)) \in \mathcal{F}^{2}(U) \times \mathcal{F}^{2}(U): A=C, B \subseteq D\right\}$ and $C_{4}=\left\{(x, y) \in \mathbb{R}^{2}: x \geq y\right\}$. (Monotonicity wrt inclusion (a))

- The interval-extension of $I\left(P^{1}\left(\mathcal{F}_{5}, C_{4}\right)\right)$, where $\mathcal{F}_{5}=$ $\left\{((A, B),(C, D)) \in \mathcal{F}^{2}(U) \times \mathcal{F}^{2}(U): B=D, A \supseteq\right.$ $C\}$. (Monotonicity wrt inclusion (b))

- $s$ satisfies $I_{G_{2}^{1}, G_{2}^{2}}\left(P^{1}\left(\mathcal{F}_{6}, C_{1}\right)\right)$, where $\mathcal{F}_{6}=$ $\left\{((A, B),(C, D)) \in \mathcal{F}^{2}(U) \times \mathcal{F}^{2}(U): A=C^{c}, B=\right.$ $\left.D^{c}\right\}$. (Complement)

At the beginning of this section, we referred to three different constructive methods of set-valued generalizations introduced in [11], respectively called the set-valued, the maxmin and the max-min-varied extension. For an arbitrary measure $f: \mathcal{F}^{k}(U) \rightarrow \mathbb{R}$, each of the three extensions is based on a concrete transformation of $k$-tuples of interval-valued fuzzy sets into collections of $k$-tuples of fuzzy sets. Let us consider a particular transformation $\mathcal{G}: \operatorname{IVF} S^{k}(U) \rightarrow \wp\left(\mathcal{F}^{k}(U)\right)$. The extreme points (infimum and supremum) of the $\mathcal{G}$-interval valued extension of $f$ are calculated as follows:

$$
\underline{[f]}(A)=\inf _{X \in \mathcal{G}(A)} f(X) \text { and } \overline{[f]}(A)=\sup _{X \in \mathcal{G}(A)} f(X) .
$$

The three constructive methods considered in [11] (encompassing many set-valued constructions from the literature) correspond to the three following transformations $\mathcal{G}_{i}$ : $I V F S^{k}(U) \rightarrow \wp\left(\mathcal{F}^{k}(U)\right)$ :

- $\mathcal{G}_{1}(A)=\left\{X \in \mathcal{F}^{k}(U): \underline{A} \subseteq X \subseteq \bar{A}\right\}$ (set-valued extension).

- $\mathcal{G}_{2}(A)=\left\{X \in \mathcal{F}^{k}(U): X=\underline{A}\right.$ or $\left.X=\bar{A}\right\}$ (max-min extension).

- $\mathcal{G}_{3}(A)=\left\{X \in \mathcal{F}^{k}(U): X(x) \in\{\underline{A}(x), \bar{A}(x)\}, \forall x \in\right.$ $U\}$ (max-min-varied extension).

Now consider a measure $f$ satisfying an arbitrary notion $N$. We can provide sufficient conditions in order to guarantee that the $\mathcal{G}$-extension of $f$ satisfies the interval extension of $\boldsymbol{N}$. The proof of the following proposition is immediate.

Proposition 1: Consider a specific transformation $\mathcal{G}$ : $\operatorname{IVFS} S^{k}(U) \rightarrow \wp\left(\mathcal{F}^{k}(U)\right)$ Suppose the infimum and supremum of Eq. 2 are attainable (i.e they respectively coincide with the minimum and the maximum), and and furthermore the following conditions hold:

$$
\begin{aligned}
&\left(G^{1}\left(A_{1}\right), \ldots, G^{1}\left(A_{n}\right)\right) \in \mathcal{D} \text { and } \\
&\left(G^{2}\left(A_{1}\right), \ldots, G^{2}\left(A_{n}\right)\right) \in \mathcal{D} \Leftrightarrow \\
&\left(\left(A_{1}\right)_{*}, \ldots,\left(A_{n}\right)_{*}\right) \in \mathcal{D} \text { and }\left(\left(A_{1}\right)^{*}, \ldots,\left(A_{n}\right)^{*}\right) \in \mathcal{D},
\end{aligned}
$$

$$
\begin{aligned}
&\left(G^{1}\left(A_{1}\right), \ldots, G^{1}\left(A_{n}\right)\right) \in \mathcal{F} \text { and } \\
&\left(G^{2}\left(A_{1}\right), \ldots, G^{2}\left(A_{n}\right)\right) \in \mathcal{F} \Rightarrow \\
&\left(\left(A_{1}\right)_{*}, \ldots,\left(A_{n}\right)_{*}\right) \in \mathcal{F} \text { and }\left(\left(A_{1}\right)^{*}, \ldots,\left(A_{n}\right)^{*}\right) \in \mathcal{F},
\end{aligned}
$$

where $A_{*}$ and $A^{*}$ respectively denote $A_{*}=$ $\arg \min _{X \in \mathcal{G}(A)} f(X)$ and $A^{*}=\arg \max _{X \in \mathcal{G}(A)} f(X)$, for all $A \in I V F S^{k}(U)$. Then, if $f$ satisfies the property of type $1, \boldsymbol{P}=P^{1}(\mathcal{D}, \mathcal{F}, C)$, then the $\mathcal{G}$-set valued extension of $f$ satisfies the interval extension of $\boldsymbol{P}, S(\boldsymbol{P})$.
Analogously, if the implication in Equation 4 is replaced by an equivalence, and $f$ satisfies a property of type $2 \boldsymbol{P}^{\prime}$, then the $\mathcal{G}$-interval-valued extension of $f$ satisfies the set-valued extension of $\boldsymbol{P}^{\prime}$.

We can see a result proved by Galar et al. in [18] as an illustration of Proposition 1. In fact, they prove that the maxmin-valued extension of a similarity measure between fuzzy sets satisfies Definition 1, i.e., satisfies the interval extension of the original notion. More concretely, suppose that $s$ is a similarity measure and define $[\underline{s}, \bar{s}]$ as follows:

$$
\underline{s}(\boldsymbol{A}):=\min _{X \in \mathcal{S}(\boldsymbol{A})} s(X), \bar{s}(\boldsymbol{A}):=\max _{X \in \mathcal{S}(\boldsymbol{A})} s(X),
$$

where $\mathcal{S}(\boldsymbol{A})=\left\{X \quad \in \quad \mathcal{F}^{k}(U) \quad: \quad X(x) \quad \in\right.$ $\{\underline{\boldsymbol{A}}(x), \overline{\boldsymbol{A}}(x)\}, \quad \forall x \in U\}$. They prove that $[\underline{s}, \bar{s}]$ : $I V F S^{2}(U) \rightarrow \mathbb{I}([0,1])$ satisfies the interval extension of the original notion. In fact, their proof is basically devoted to show that Equations 3 and 4 are satisfied for every property of similarity measures (symmetry, min. value, max. value, monotonicity wrt inclusion and complement), where a particular instance of $G^{1}, G^{2}, \mathcal{D}$ and $\mathcal{F}$ is considered.

\section{SCALAR EXTENSIONS}

A general axiomatic definition has been proposed in the last section. It has been shown to encompass some existing axiomatic definitions generalizing some FS notions to the case of IVF and IF sets and leading to set-valued measurements. Let us nevertheless notice that the literature about set-valued generalizations of FS measures is not very vast. Furthermore, most of those generalizations deal with constructive definitions (see [11] for a review and a formal analysis of them) instead of axiomatic definitions like the ones considered in the previous section.

Now we will review another much broader category of extensions from the literature, where the images of the corresponding extension are numbers instead of intervals. We can again distinguish between the so-called constructive and the axiomatic definitions. Instances of the first group of definitions are the different variants of the notion of measure of "similarity" introduced in [15], [24], [26], [27], [31], [44]), and of the notion of "distance" measure (see [22], [25], [39], [41]), the "average cardinality" introduced by Wu and Mendel in [42], or the "correlation" between two IF sets given in [17], among many others. In all the above cases, the authors propose an extension of an existing formula and prove some properties of the new function.

But there are other papers in the literature where the authors propose axiomatic definitions of the new extended concepts. We have reviewed several examples of this kind of "axiomatic extension" in different contexts, and we have observed some formal common features that allow us to conduct a generalized analysis. Let us start by providing several examples of axiomatic extensions, as an introduction to this encompassing approach. This is the case of the axiomatic definitions of similarity between IF sets by Denfeng-Chuntiang [15] and Zhang [48], the notion distance between IF sets by Zhang [48] or the notion of divergence by I. Montes et al. [32]. We will recall the three of them. The following definition 
given by Denfeng and Chuntiang [15] deals with the notion of "similarity" between two intuitionistic fuzzy sets. (We will state a formally equivalent definition in the context of intervalvalued fuzzy sets).

Definition 7: $s: \operatorname{IVF} S^{2}(U) \rightarrow \mathbb{R}$ is a similarity measure if it satisfies the following properties:

- $\mathrm{S}(\operatorname{Sim} 1) .-0 \leq s(A, B) \leq 1, \forall A, B \in I V F S(U)$.

- $\mathrm{S}(\mathrm{Sim} 2) .-A=B \Leftrightarrow s(A, B)=1$.

- $\mathrm{S}(\operatorname{Sim} 3)$.- $s(A, B)=s(B, A), \forall A, B \in I V F S(U)$.

- S(Sim 4).- If $A \subseteq_{L} B \subseteq_{L} C$ then $s(A, C) \leq s(A, B)$, where $\subseteq_{L}$ denotes the lattice inclusion.

- S(Sim 5).- If $A \subseteq_{L} B \subseteq_{L} C$ then $s(A, C) \leq s(B, C)$.

The following notion of "distance" between two intervalvalued fuzzy sets has been introduced by Zhang et al. in [48] and generalizes a previous definition given by Liu [29] in the context of fuzzy sets. It is dual to another variant of the notion of "similarity" also provided by Zhang et al. in [48].

Definition 8: $d: I V F S^{2}(U) \rightarrow \mathbb{R}$ is a distance measure if it satisfies the following properties:

- S(DP1).- $d(A, B)=d(B, A), \forall A, B \in I V F S(U)$.

- S(DP2).- $d(A, B)=1 \Leftrightarrow B=A^{c}, A \in \wp(U)$.

- $\mathrm{S}(\mathrm{DP} 3) .-A=B \Leftrightarrow d(A, B)=0$.

- S(DP4).- If $A \subseteq_{L} B \subseteq_{L} C$ then $d(A, C) \geq d(A, B)$.

- S(DP5).- If $A \subseteq_{L} B \subseteq_{L} C$ then $d(A, C) \geq d(B, C)$.

The following definition generalizes the notion of divergence between fuzzy sets initially introduced by Montes et al. in [32]. It has been stated in [32] within the context of intuitionistic fuzzy sets and we will provide a formally equivalent definition for interval-valued fuzzy sets.

Definition 9: $D_{I F}: I V F S^{2}(U) \rightarrow \mathbb{R}$ is an IVFS-divergence if it satisfies the following properties:

- $\mathrm{S}\left(\right.$ Div1).- $D_{I F}(A, B)=D_{I F}(B, A), \quad \forall A, B \in$ IVFS $(U)$.

- $\mathrm{S}$ (Div2).- $A=B \Rightarrow D_{I F}(A, B)=0$.

- $\mathrm{S}$ (Div3).- $D_{I F}(A \cap C, B \cap C) \leq D_{I F}(A, B), \forall A, B, C \in$ IVFS $(U)$.

- $\mathrm{S}$ (Div4).- $D_{I F}(A \cup C, B \cap C) \leq D_{I F}(A, B), \forall A, B, C \in$ $\operatorname{IVFS}(U)$.

We can detect common features in the above definitions. All of them link the properties of the original definition (the particular formulation for pairs of fuzzy sets) to the properties of the extended one, for pairs of interval-valued fuzzy sets or intuitionistic fuzzy sets. In fact, they take each property in the original definition and extend it according to the general procedure that we will describe in Definition 11. We previously need to provide an auxiliary definition:

Definition 10: Let us consider two collections of tuples of fuzzy sets $\mathcal{D}, \mathcal{F} \subseteq \mathcal{F}^{k}(U) \times \stackrel{(n)}{.} \times \mathcal{F}^{k}(U)$ and a set $C \subseteq \mathbb{R}^{n}$. Let us consider the properties $\boldsymbol{P}=P^{1}(\mathcal{D}, \mathcal{F}, C)$ and $\boldsymbol{P}^{\prime}=$ $P^{2}(\mathcal{D}, \mathcal{F}, C)$. Let us consider a pair of mappings $G^{1}, G^{2}$ : $I V F S^{k}(U) \rightarrow \mathcal{F}^{k}(U)$.

- We say that $f: I V F S^{k}(U) \rightarrow \mathbb{R}$ satisfies the $\left(G^{1}, G^{2}\right)$ scalar-extension of $\boldsymbol{P}, S_{G^{1}, G^{2}}(\boldsymbol{P})$ if :

$$
\begin{array}{r}
\left(G^{1}\left(\boldsymbol{A}_{\mathbf{1}}\right), \ldots, G^{1}\left(\boldsymbol{A}_{\boldsymbol{k}}\right)\right),\left(G^{2}\left(\boldsymbol{A}_{\mathbf{1}}\right), \ldots, G^{2}\left(\boldsymbol{A}_{\boldsymbol{k}}\right)\right) \in \mathcal{F} \\
\Rightarrow\left(f\left(\boldsymbol{A}_{\mathbf{1}}\right), \ldots, f\left(\boldsymbol{A}_{\boldsymbol{n}}\right)\right) \in C .
\end{array}
$$

- We say that $f: I V F S^{k}(U) \rightarrow \mathbb{R}$ satisfies the $\left(G^{1}, G^{2}\right)$ scalar-extension of $\boldsymbol{P}^{\prime}, S_{G^{1}, G^{2}}\left(\boldsymbol{P}^{\prime}\right)$ if:

$$
\begin{array}{r}
\left(G^{1}\left(\boldsymbol{A}_{\mathbf{1}}\right), \ldots, G^{1}\left(\boldsymbol{A}_{\boldsymbol{k}}\right)\right),\left(G^{2}\left(\boldsymbol{A}_{\mathbf{1}}\right), \ldots, G^{2}\left(\boldsymbol{A}_{\boldsymbol{k}}\right)\right) \in \mathcal{F} \\
\Leftrightarrow\left(f\left(\boldsymbol{A}_{\mathbf{1}}\right), \ldots, f\left(\boldsymbol{A}_{\boldsymbol{n}}\right)\right) \in C .
\end{array}
$$

Based on the above definitions, we can describe the general procedure that encompasses Definitions 7,8 and 9, among many others:

Definition 11: Let us consider the notion $\mathbf{N}=$ $N\left(\boldsymbol{P}_{1}, \ldots, \boldsymbol{P}_{l}\right)$ where $\boldsymbol{P}_{i}$ is either a property of type 1 or of type 2 , for all $i=1, \ldots, l$. We say that $S: I V F S^{k}(U) \rightarrow \mathbb{R}$ satisfies the scalar-extension of $\mathbf{N}, S(\mathbf{N})$, if it satisfies the scalar-extended properties $S\left(\boldsymbol{P}_{1}\right), \ldots, S\left(\boldsymbol{P}_{l}\right)$.

The following example shows how the notion of similarity measure recalled in Definition 7 can be seen as a particular case of Definition 11.

Example 3: Let us consider again the notion of similarity measure recalled in Example 1. There we showed that the five characterizing properties Sim 1 to Sim 5 of similarity measures can be alternatively formulated as follows:

- Sim 1) $s$ satisfies $P^{1}\left(\mathcal{F}_{1}, C_{1}\right)$ with $\mathcal{F}_{1}=\mathcal{F}^{2}(U)$ and $C_{1}=[0,1]$.

- Sim 2) $s$ satisfies $P^{2}\left(\mathcal{F}_{2}, C_{2}\right)$ with $\mathcal{F}_{2}=\{(X, X): X \in$ $\mathcal{F}(U)\}$ and $C_{2}=\{1\}$.

- $\operatorname{Sim}$ 3) $s$ satisfies $P^{1}\left(\mathcal{F}_{3}, C_{3}\right)$, where $\mathcal{F}_{3}=$ $\left\{(X, Y),(Z, T) \in \mathcal{F}^{2}(U) \times \mathcal{F}^{2}(U): X=T, Y=Z\right\}$ and $C_{3}=\{(x, x): x \in[0,1]\}$.

- Sim 4) $s$ satisfies $P^{1}\left(\mathcal{F}_{4}, C_{4}\right)$ with $\mathcal{F}_{4}=$ $\left\{(X, Y),(Z, T) \in \mathcal{F}^{2}(U) \times \mathcal{F}^{2}(U): X=Z, Y \subseteq Z\right\}$ and $C_{4}=\left\{(x, y) \in \mathbb{R}^{2}: x \geq y\right\}$.

- $\operatorname{Sim}$ 5) $s$ satisfies $P^{1}\left(\mathcal{F}_{5}, C_{4}\right)$ with $\mathcal{F}_{5}=$ $\left\{(X, Y),(Z, T) \in \mathcal{F}^{2}(U) \times \mathcal{F}^{2}(U): X \subseteq Z, Y=Z\right\}$.

According to this alternative, but equivalent formulation, we can easily see that each of the properties denoted as " $\mathrm{S}$ (Sim i)" in Definition 7 is in fact the scalar-extension of "Sim $i$ ", for every $i=1, \ldots, 5$ in accordance with Definition 10 . Therefore Definition 7 is an extension of the original definition of similarity measure between fuzzy sets in accordance to the general procedure described in Definition 11.

We can analogously prove that Definitions 8 and 9 also follow the general procedure described in Definition 11. These three definitions are just three options among all the variants of the notions of "similarity", "similitude", "resemblance", "dissimilarity", "distance", etc reviewed in [12]. All the notions reviewed there could be easily generalized to the case of interval-valued fuzzy sets or intuitionistic fuzzy sets according to the general procedure proposed in Definition 11. Furthermore, we may ask ourselves whether the formal relations connecting those different notions studied in [12] are also satisfied in this more general context of intervalvalued and intuitionistic fuzzy sets. The answer is based on the following formal result, whose proof is straightforward:

Proposition 2: Let us consider a collection of properties $\quad \mathbf{P}^{i_{1}}\left(\mathcal{D}_{1}, \mathcal{F}_{1}, C_{1}\right), \ldots, \mathbf{P}^{i_{l}}\left(\mathcal{D}_{l}, \mathcal{F}_{l}, C_{l}\right)$. Let $S\left(\mathbf{P}^{i_{1}}\left(\mathcal{D}_{1}, \mathcal{F}_{1}, C_{1}\right)\right), \ldots, S\left(\mathbf{P}^{i_{l}}\left(\left(\mathcal{D}_{l}, \mathcal{F}_{l}, C_{l}\right)\right)\right.$ denote their respective scalar extensions. If:

- $\min \left\{i_{1}, \ldots, i_{l-1}\right\} \geq i_{l}$. 


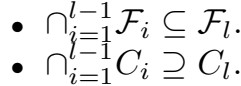

Then:

- $\mathbf{P}^{i_{1}}\left(\mathcal{F}_{1}, C_{1}\right) \wedge \ldots \wedge \mathbf{P}^{i_{l-1}}\left(\mathcal{F}_{l-1}, C_{l-1}\right) \Rightarrow \mathbf{P}^{i_{l}}\left(\mathcal{F}_{l}, C_{l}\right)$.

- $S\left(\mathbf{P}^{i_{1}}\left(\mathcal{F}_{1}, C_{1}\right)\right) \wedge \ldots \wedge S\left(\mathbf{P}^{i_{l-1}}\left(\mathcal{F}_{l-1}, C_{l-1}\right)\right) \Rightarrow$ $S\left(\mathbf{P}^{i_{l}}\left(\mathcal{F}_{l}, C_{l}\right)\right)$.

The above result may help to prove some implication relations between different variants of related notions, such as those mentioned above (similarity, similitude, resemblance, etc). Most of those notions involve properties of type 1 and type 2, and the proofs about relations of implication between different notions are based on the study about some inclusion relations between the sets $\mathcal{F}_{i}$ and $C_{i}$ that characterize the involved properties. In all those cases, and taking into account the above result, those proofs regarding the corresponding inclusion relations can be directly used in order to extend those existing implication results to the more general context of intuitionistic fuzzy sets / interval-valued fuzzy sets. Of course, the above result is not restricted to the study of different variants of the notions of "degree of equality" and "degree of inequality" between IVF or IF sets. Thus, a detailed study about the implication relations between those notions falls out of the scope of this paper. An exhaustive study about those implication relations in the context of fuzzy sets can be found in [12], [14]. On the other hand, some specific proofs about the relation between the notion of divergence measures and other notions in the general context of IF sets can be found in [32].

The above references [12], [14], [32] also contain counterexamples showing that some implications do not hold. In [12], [14], an exhaustive list of examples in order to provide an overall picture, within the context of fuzzy sets, about what notions are stronger than others is provided. On the other hand, one specific example showing that one of those implications does not either hold in the general context of IF sets is given in [32]. Up to this point, we may ask ourselves what happens in general: If a notion $\mathbf{N}$ does not imply another one $\mathbf{N}^{\prime}$ within the context of fuzzy sets, is it possible that $S(\mathbf{N})$ (the scalar extension of $\mathbf{N}$ ) implies $S\left(\mathbf{N}^{\prime}\right)$ ? The answer is no, as we prove below:

Proposition 3: Suppose that there exists a mapping $f$ : $\mathcal{F}^{k}(U) \rightarrow \mathbb{R}$ satisfying notion $\boldsymbol{N}$ but not satisfying notion $\boldsymbol{N}^{\prime}$. Then there exists a mapping $S(f): I V F S^{k}(U) \rightarrow \mathbb{R}$ satisfying the scalar extension of $\mathbf{N}, S(\mathbf{N})$, but not satisfying $S\left(\mathbf{N}^{\prime}\right)$.

Proof: Consider $S(f): I V F S^{k}(U) \rightarrow \mathbb{R}$ defined as $S(f)(A)=f(\underline{A})$. It satisfies $S(N)$ but does not satisfy $S\left(N^{\prime}\right)$.

According to Proposition 3, any counterexample showing the lack of implication between two different notions can be used as an evidence about the lack of implication between their respective scalar extensions, and explicit examples in this general framework would be unnecessary. As an example of an application of the above result, we observe that all the counterexamples considered in [12] and [14] can be easily adapted to the contexts of IVF and IF sets.

\section{A. Construction methods of scalar extensions}

As we have already mentioned, many works in the recent literature (see [15], [22], [24]-[27], [39], [41], [44] for instance) provide concrete formulas in order to construct specific (parametric families of) measures for IFS / IVFS. When the properties of the corresponding measures can be expressed either as properties of type 1 or of type 2 , we can systematize this kind of constructions. Propositions 4, 6 and 7 provide three different generalized methods of construction.

1) First Method: functions of the max-min set-valued extension: Proposition 4 provides the first general method. The proof is straightforward.

Proposition 4: Let us suppose that $M$ : $\mathcal{F}^{k}(U) \rightarrow \mathbb{R}$ satisfies the notion $\mathbf{N}=$ $N\left(P^{1}\left(\mathcal{D}_{1}, \mathcal{F}_{1}, C_{1}\right), \ldots, P^{1}\left(\mathcal{D}_{m}, \mathcal{F}_{m}, C_{m}\right)\right.$, $\left.P^{2}\left(\mathcal{D}_{1}^{\prime}, \mathcal{F}_{1}^{\prime}, C_{1}^{\prime}\right), \ldots, P^{2}\left(\mathcal{D}_{m^{\prime}}^{\prime}, \mathcal{F}_{l}^{\prime}, C_{m^{\prime}}^{\prime}\right)\right)$.

Let us construct the function $S_{h}(M): I V F S^{k}(U) \rightarrow \mathbb{R}$ defined as

$$
S_{h}(M)(A)=h(M(\underline{A}), M(\bar{A})) .
$$

Then $S_{h}(M)$ satisfies the extended notion $S(\mathbf{N})$ if and only if $h: \mathbb{R}^{2} \rightarrow \mathbb{R}$ satisfies the following conditions:

$$
\begin{array}{r}
\left(x_{1}, \ldots, x_{n}\right) \in C_{i},\left(y_{1}, \ldots, y_{n}\right) \in C_{i} \Rightarrow \\
\left(h\left(x_{1}, y_{1}\right), \ldots, h\left(x_{n}, y_{n}\right)\right) \in C_{i}, i=1, \ldots, k \\
\text { and }\left(x_{1}, \ldots, x_{n}\right) \in C_{j}^{\prime},\left(y_{1}, \ldots, y_{n}\right) \in C_{j}^{\prime} \Leftrightarrow \\
\left(h\left(x_{1}, y_{1}\right), \ldots, h\left(x_{n}, y_{n}\right)\right) \in C_{j}^{\prime}, j=1, \ldots, k^{\prime} .
\end{array}
$$

From now on, we will refer to $S_{h}(M)$ as the extreme-based scalar extension of $M$.

Example 4: Let us suppose that $M: \mathcal{F}^{k}(U) \rightarrow \mathbb{R}$ satisfies the following condition, for a certain $\mathcal{F} \subseteq \mathcal{F}^{k}(U) \times \mathcal{F}^{k}(U)$ :

$$
\left[\left(A_{1}, A_{2}\right) \in \mathcal{F} \Rightarrow M\left(A_{1}\right) \geq M\left(A_{2}\right)\right]
$$

The above condition is a property of type 1 , since it can be alternatively expressed as follows:

$$
\left[\left(A_{1}, A_{2}\right) \in \mathcal{F} \Rightarrow\left(M\left(A_{1}\right), M\left(A_{2}\right)\right) \in C\right],
$$

where $\left.C=\left\{(x, y) \in \mathbb{R}^{2}: x \geq y\right\}\right]$. Let us call it $\boldsymbol{P}$. Then the mapping $S_{h}(M): I V F S^{k}(U) \rightarrow \mathbb{R}$ defined as $S_{h}(M)(A)=$ $h(M(\underline{A}), M(\bar{A}))$ satisfies the extended property $S(\boldsymbol{P})$ if and only if $h: \mathbb{R}^{2} \rightarrow \mathbb{R}$ is increasing in both components.

The proof of this result is a consequence of the last proposition and the fact that, if $C=\left\{(x, y) \in \mathbb{R}^{2}: x \geq y\right\}$ then Equation 9 (below) holds if and only if $h$ is an increasing function.

$$
\left[\left(x_{1}, x_{2}\right) \in C,\left(y_{1}, y_{2}\right) \in C \Rightarrow\left(h\left(x_{1}, y_{1}\right), h\left(x_{2}, y_{2}\right)\right) \in C\right]
$$

Example 5: Let us now suppose that $M: \mathcal{F}^{k}(U) \rightarrow \mathbb{R}$ satisfies the following condition for a certain $\mathcal{F} \subseteq \mathcal{F}^{k}(U)$ and a certain $c \in \mathbb{R}$ :

$$
A \in \mathcal{F} \Rightarrow f(A)=c .
$$


The above condition can be seen as a property of type 1 (the property $\left.\mathbf{P}=P^{1}(\mathcal{F},\{c\})\right)$. Then, according to the above result, for any $h: \mathbb{R}^{2} \rightarrow \mathbb{R}$ such that $h(c, c)=c$, the mapping $S_{h}(M)=h(M(\underline{A}), M(\bar{A}))$ satisfies the extended property $S(\mathbf{P})$.

As we have recalled at the beginning of this subsection, many authors in the literature provide concrete formulas of measures in the context of IFS, and they prove that those measures satisfy certain properties (in accordance with some specific axiomatic definition). Many of those particular formulations match the general procedure described in Proposition 4. Some examples of those are the normalized Hamming distance proposed by Burillo and Bustince in [5], and also mentioned by Atanassov [4] and Grzegorzewski [22], or the notion of similarity introduced by Mitchell in [31].

The following example uses the particularizations of Proposition 4 given in Examples 4 and 5 in order to construct measures of similarity and measures of divergence between IFS based on their respective analogous for the particular context of fuzzy sets.

Example 6: The first part of this example shows how to construct measures of similarity between IVF sets in accordance with Definition 7. The second part shows how to construct measures of divergence between IVS sets (or formally equivalently, between intuitionistic fuzzy sets).

- Let us suppose that $s: \mathcal{F}^{2}(U) \rightarrow \mathbb{R}$ satisfies axioms Sim 1-Sim 5 enumerated in Example 1. Let us suppose that $h:[0,1] \times[0,1] \rightarrow[0,1]$ satisfies the following conditions:

- $h(0,0)=0$

- $h(1,1)=1$

- $h$ is increasing in both components (but not necessarily strictly increasing).

Then, the mapping $S(s): I V F S^{2}(U)$ defined as

$$
S(s)(A, B)=h(s(\underline{A}, \underline{B}), s(\bar{A}, \bar{B}))
$$

satisfies the extended notion recalled in Definition 7. We can find in [31] several examples of extended similarity measures that follow this formulation.

- Let $d: \mathcal{F}^{2}(U) \rightarrow \mathbb{R}$ be a divergence measure according to [33]. If $h: \mathbb{R} \times \mathbb{R} \rightarrow \mathbb{R}$ satisfies the following properties:

- $h(0,0)=0$

- $h$ is increasing in both components (but not necessarily strictly increasing).

Then $S(d): I V F S^{2}(U) \rightarrow \mathbb{R}$ defined as

$$
S(d)(A, B)=h(d(\underline{A}, \underline{B}), d(\bar{A}, \bar{B}))
$$

satisfies the extended notion recalled in Definition 9. This result has been proved by I. Montes et al. in [32]. If we take the original properties of divergence measures between fuzzy sets into account, the proof is a direct consequence of Proposition 4.

The general construction method considered in this subsection generalizes many proposals from the recent literature, as we have recalled in Example 6. In many of those cases the mapping $h$ is symmetric. Under this constraint, $h(x, y)$ coincides with $h(\min \{x, y\}, \max \{x, y\})$ and therefore $S_{h}(M)=h(M(\underline{A}), M(\bar{A}))$ can be alternatively expressed as

$$
S_{h}(M)=h(\min \{M(\underline{A}), M(\bar{A})\}, \max \{M(\underline{A}), M(\bar{A})\}) .
$$

Thus, under this constraint, the extreme-based scalar extension is calculated as a function of the extreme points, $\min \{M(\underline{A}), M(\bar{A})\}$ and $\max \{M(\underline{A}), M(\bar{A})\}$, of the maxmin extension considered in [11]. Some authors have argued that scalar extensions are less informative than setvalued extensions of different measures. In fact, for the kind of construction considered here, the process of selection of a real number as a function of the pair $(\min \{M(\underline{A}), M(\bar{A})\}, \max \{M(\underline{A}), M(\bar{A}))$, incurs in a loss of information in favor of a precisiation of the value of $M(A)$.

The following method provides an alternative construction that can be seen as a function of the max-min extension. It coincides with the extremes-based construction when $h$ is symmetric. But in the general case, it does not require $M(\underline{A})$ and $M(\bar{A})$ to be treated symmetrically.

Proposition 5: Let us suppose that $M$ : $\mathcal{F}^{k}(U) \rightarrow \mathbb{R}$ satisfies the notion $\mathbf{N}=$ $N\left(P^{1}\left(\mathcal{F}_{1}, C_{1}\right), \ldots, P^{1}\left(\mathcal{F}_{k}, C_{k}\right), P^{2}\left(\mathcal{F}_{1}^{\prime}, C_{1}^{\prime}\right), \ldots, P^{2}\left(\mathcal{F}_{l}^{\prime}, C_{l}^{\prime}\right)\right)$, for some specific collections of tuples of fuzzy sets $\mathcal{F}_{1}, \ldots, F_{k}, \mathcal{F}_{1}^{\prime}, \ldots, \mathcal{F}_{l}^{\prime}$ and some specific collections of vectors $C_{1}, \ldots, C_{k}, C_{1}^{\prime}, \ldots, C_{l}^{\prime}$.

Let us construct the function $S_{h}^{\prime}(M): \operatorname{IVF} S^{k}(U) \rightarrow \mathbb{R}$ defined as

$$
S_{h}^{\prime}(M)(A)=h(\min \{M(\underline{A}), M(\bar{A})\}, \max \{M(\underline{A}), M(\bar{A})\}) .
$$

Then $S_{h}^{\prime}(M)$ satisfies the extended notion $S(\mathbf{N})$ if and only if the mapping $h:\left\{(x, y) \in \mathbb{R}^{2}: x \leq y\right\} \rightarrow \mathbb{R}$ satisfies the following conditions:

$$
\begin{array}{r}
\left(x_{1}, \ldots, x_{n}\right) \in C_{i},\left(y_{1}, \ldots, y_{n}\right) \in C_{i} \Rightarrow \\
h\left(x_{1} \wedge y_{1}, x_{1} \vee y_{1}\right), \ldots, h\left(x_{n} \wedge y_{n}, x_{n} \vee y_{n}\right) \in C_{i}, \\
i=1, \ldots, k \\
\text { and } \\
\left(x_{1}, \ldots, x_{n}\right) \in C_{j}^{\prime},\left(y_{1}, \ldots, y_{n}\right) \in C_{j}^{\prime} \Leftrightarrow \\
h\left(x_{1} \wedge y_{1}, x_{1} \vee y_{1}\right), \ldots, h\left(x_{n} \wedge y_{n}, x_{n} \vee y_{n}\right) \in C_{i}, \\
i=1, \ldots, k^{\prime},
\end{array}
$$

(where $\wedge$ and $\vee$ respectively denote the minimum and the maximum). From now on, we will refer to $S_{h}^{\prime}(M)$ as the $\max$ min-based scalar extension of $M$.

If the mapping $h$ satisfies the following inequalities:

$$
\min \{a, b\} \leq h(a, b) \leq \max \{a, b\}, \forall(a, b),
$$

then the max-min-based scalar construction can be regarded as a procedure that selects a somehow representative point inside the interval determined by the max-min extension.

The next subsection presents a parallel construction where a representative point of the max-min-varied extension is selected. 
2) Second method: extension of T-internal measures: The method proposed in this section needs the original measure $M$ to satisfy a certain property of "additivity" introduced in Definition 13. Let us first give an auxiliary definition.

Definition 12: Let us consider a universe $U$ and an arbitrary element $x^{*} \in U$. Given a function $M: \mathcal{F}(U)^{k} \rightarrow$ $\mathbb{R}$ let $M_{x^{*}}$ denote the mapping $M_{x^{*}}\left(A^{1}, \ldots, A^{k}\right)=$ $M\left(\left(A^{1}\right)_{x^{*}}, \ldots,\left(A^{k}\right)_{x^{*}}\right)$ where

$$
A_{x^{*}}(x)= \begin{cases}A\left(x^{*}\right) & \text { if } x=x^{*} \\ 0 & \text { otherwise. }\end{cases}
$$

$M_{x^{*}}$ is called the $x^{*}$-restriction of $M$.

Definition 13: Consider an aggregation operator $T$. A notion $\mathbf{N}$ is said to be $T$-internal if the following implications hold:

- If $M$ satisfies $\mathbf{N}$ then its $x$-restriction also satisfies $\mathbf{N}$ for every $x \in U$.

- If $M_{1}, \ldots, M_{n}$ satisfy the notion $\mathbf{N}$ then $T\left(M_{1}, \ldots, M_{n}\right)$ also satisfies $\mathbf{N}$, for every $n \in \mathbb{N}$.

The following result describes a second construction method. This result guarantees that, if $M$ satisfies a certain $T$-internal notion $\mathbf{N}$, then the mapping $S^{\prime \prime}(M)$ fulfills the extended notion $S(\mathbf{N})$.

Proposition 6: Consider the finite universe $U=$ $\left\{x_{1}, \ldots, x_{n}\right\}$. Suppose that $M$ satisfies the $T$ - internal notion N. Let $S_{h}^{\prime \prime}(M)$ be defined as follows:

$$
\begin{array}{r}
S_{h}^{\prime \prime}(M)(A)= \\
T\left(h\left(M_{x_{1}}(\underline{A}), M_{x_{1}}(\bar{A})\right), \ldots, h\left(M_{x_{n}}(\underline{A}), M_{x_{n}}(\bar{A})\right)\right.
\end{array}
$$

Then $S_{h}^{\prime \prime}(M)$ satisfies the scalar extension $S(\mathbf{N})$ if and only if $h$ satisfies the conditions established in Equation 8.

From now on, $S_{h}^{\prime \prime}(M)$ will be called the T-scalar extension of $M$.

Some distance measures, based on the so-called "normalized Hamming distance" ( [4], [5], [22]), or other distances based on the Hausdorff metric [22], as well as the concepts of implication operations and strong equality indices in intervalvalued fuzzy sets ( [46]) can be seen as some examples of this general construction.

This scalar extension is quite related to the (set-valued) maxmin-varied extension. In fact, many constructions of measures within the fuzzy sets literature, such as [7], [8], lead to measures that are generated on the basis of some aggregation operator, in the sense that they can be written as follows:

$$
M(A)=T\left(M_{x_{1}}(A), \ldots, M_{x_{n}}(A)\right), \forall A \in I V F S(U),
$$

for some aggregation operator $T$. If furthermore $h$ satisfies Equation 11, then due to the monotonicity of $T$, the value $S_{h}^{\prime \prime}(M)(A)$ belongs to the max-min-varied interval $\left[\inf _{X \in \mathcal{G}_{3}(A)} M(X), \sup _{X \in \mathcal{G}_{3}(A)} M(X)\right]$.

3) Third method: The third construction is somehow different from the previous construction proposed in Subsections IV-A1 and IV-A2. In the first case, we picked a value out of the interval determined by the max-min extension. In the second case, we repeated the same strategy for every $x \in U$ and then we "averaged" the measurement all over the elements of $U$.
Thus, in those cases, we disambiguated "at the end", i.e., after calculating the interval of measurements. Here, we start by picking a set $X$ in the class $\{X: \underline{A} \subseteq X \subseteq \bar{A}\}$ and then we calculate the value $M(X)$. Instead of "disambiguating at the end" as we do with the max-min scalar extension, (where we pick a value that is calculated as a function of the extremes of the interval determined max-min extension), we "disambiguate at the beginning".

Proposition 7: Let us suppose that $M$ : $\mathcal{F}(U) \times \stackrel{(n)}{.} \times \mathcal{F}(U) \rightarrow \mathbb{R}$ satisfies the notion $\mathbf{N}=$ $N\left(P^{1}\left(\mathcal{F}_{1}, C_{1}\right), \ldots, P^{1}\left(\mathcal{F}_{k}, C_{k}\right), P^{2}\left(\mathcal{F}_{1}^{\prime}, C_{1}^{\prime}\right), \ldots, P^{2}\left(\mathcal{F}_{l}^{\prime}, C_{l}^{\prime}\right)\right)$, for some specific collections of tuples of fuzzy sets $\mathcal{F}_{1}, \ldots, F_{k}, \mathcal{F}_{1}^{\prime}, \ldots, \mathcal{F}_{l}^{\prime}$ and some specific collections of vectors $C_{1}, \ldots, C_{k}, C_{1}^{\prime}, \ldots, C_{l}^{\prime}$.

Let us construct the function $S_{h}(M): I V F S^{k}(U) \rightarrow \mathbb{R}$ defined as

$$
S_{h}^{\prime \prime \prime}(M)(A)=M(f(\underline{A}, \bar{A})) .
$$

Then $S_{h}^{\prime \prime \prime}(M)$ satisfies the extended notion $S(\mathbf{N})$ if and only if $f: I V F S^{k}(U) \rightarrow \mathcal{F}^{k}(U)$ satisfies the following conditions:

$$
\begin{array}{r}
\left(\underline{A_{1}}, \ldots, \underline{A_{n}}\right) \in \mathcal{F}_{i},\left(\overline{A_{1}}, \ldots, \overline{A_{n}}\right) \in \mathcal{F}_{i} \Rightarrow \\
\left(f\left(\underline{A_{1}}, \overline{A_{1}}\right), \ldots, f\left(\underline{A_{n}}, \overline{A_{n}}\right)\right) \in \mathcal{F}_{i}, i=1, \ldots, k \\
\left(\underline{A_{1}}, \ldots, \underline{A_{n}}\right) \in \mathcal{F}_{i},\left(\overline{A_{1}}, \ldots, \overline{A_{n}}\right) \in \mathcal{F}_{i}^{\prime} \Leftrightarrow \\
\left.\left(\underline{f\left(\underline{A_{1}}\right.}, \overline{A_{1}}\right), \ldots, f\left(\underline{A_{n}}, \overline{A_{n}}\right)\right) \in \mathcal{F}_{i}^{\prime} .
\end{array}
$$

From now on, we will refer to $S_{h}^{\prime \prime \prime}(M)$ as the disambiguationbased scalar extension of $M$.

Examples of this construction are the measures based on the fuzzy set membership function constructed as

$$
\varphi_{A}=\frac{\underline{A}+\bar{A}}{2}
$$

and proposed by Deng [15] and Liang and Shi [28]. The normalized Hamming distance can be also seen as an example of this kind of construction.

\section{CONCLUDING REMARKS}

We have reviewed different axiomatic recent definitions in the context of IVF sets and IF sets. Many of them extend axiomatic definitions originally proposed in the context of fuzzy sets. Looking at them from a purely formal perspective, and forgetting about every concrete notion, we have observed that both, the original definitions (the ones formulated in the context of fuzzy sets) and the process of extension (from fuzzy sets to IVF sets) share certain commonalities. In fact, many of those original definitions consist of a list of requirements that a mapping $f: \mathcal{F}^{k}(U) \rightarrow \mathbb{R}$ must satisfy. Those requirements can be either formulated as properties of type 1 :

$$
\begin{gathered}
\forall\left(A_{1}, \ldots, A_{n}\right) \in \mathcal{D} \\
\left(A_{1}, \ldots, A_{n}\right) \in \mathcal{F} \Rightarrow\left(f\left(A_{1}\right), \ldots, f\left(A_{n}\right)\right) \in C
\end{gathered}
$$

or as properties of type 2 :

$$
\forall\left(A_{1}, \ldots, A_{n}\right) \in \mathcal{D},
$$




$$
\left(A_{1}, \ldots, A_{n}\right) \in \mathcal{F} \Leftrightarrow\left(f\left(A_{1}\right), \ldots, f\left(A_{n}\right)\right) \in C .
$$

(For a certain triple $\mathcal{D} \in \mathcal{F}^{k}(U) \times \ldots \times \mathcal{F}^{k}(U), \mathcal{F} \in \mathcal{F}^{k}(U) \times$ $\left.\ldots \times \mathcal{F}^{k}(U), C \in \mathbb{R}^{n}\right)$.

On the other hand, if we focus on the extension procedure that converts the original fuzzy sets' definition into a generalized one, we can observe that the process of transformation of the above properties often follows a similar pattern. We have separately studied set-valued extensions from real-valued extensions, and we have proposed two general definitions, each of them corresponding to each of those cases. Thus, we have observed that many axiomatic set-valued extensions from the literature follow the general procedure described in Definitions 5 and 6, while many scalar generalized definitions follow the procedure described in Definitions 10 and 11. Such an abstraction allows to notably organize and simplify the process of construction of new extended measures, and to note that many definitions from the literature are nothing else but particular instances of Definitions 6 or 11.

We have analyzed the three general procedures of construction of set-valued measures considered in [11] under the light of this general scheme. We have additionally proposed three different but related general procedures of constructions of scalar measures in the context of IVF sets. The six constructions encompass many different particular constructions from the literature as particular cases. Such a general framework allows us to simplify many proofs: we can find in the literature plenty of works where some particular constructive definition in the context of fuzzy sets is extended to the general context of IVF sets. The original constructive definition is known to satisfy a list of properties that can be often formulated either as properties of type 1 or type 2 . The authors prove that the extended construction satisfies a certain list of properties. Those properties very often coincide with the extended version of the original ones via Definition 5 (interval extension) or Definition 10 (scalar extension), and furthermore the extended construction proposed by the authors follows some of our six procedures. According to the results included in this paper, the (sometimes long) proofs provided in some papers in order to check that a particular construction satisfies the list of extended properties could be substantially simplified.

Notwithstanding, with respect to the second case (realvalued extensions of the FS concept) we should notice that some well known definitions from the literature escape from the general formulation provided in Definition 11. To give an example, the notion of entropy of IF sets introduced by Szmidt and Kacprzyk in [40] and adapted to IVF sets by Zeng and $\mathrm{Li}$ [47], extends the well-known notion of entropy of a fuzzy set originally introduced by De Luca and Termini [30] cannot be formulated in terms of Definition 11. The authors base the interpretation of their extension on a geometric representation of IF sets. According to their interpretation, the entropy measures the whole missing information which may be necessary to classify the points as elements either with full membership or full non-membership degree. It is not only related to the degrees of fuzziness of both extremes $A$ and $\bar{A}$ but also to the hesitation degree of the IVF set. In fact, if $\underline{A}$ is less fuzzy than $\underline{B}$ and $\bar{A}$ is less fuzzy than
$\bar{B}$ (in accordance to De Luca and Termini's partial ordering) and furthermore, $H(A)(x)=\bar{A}(x)-\underline{A}(x)$ is greater than or equal to $H(B)(x)$, for every $x \in U$, then $A$ is less fuzzy than $B$, in accordance to Szmidt-Kacprzyk axiomatic definition. This axiomatic definition is not an isolate example. Other parametric families of extended measures in the literature also take into account the hesitation degree. In the near future, we plan to analyze where there are or not formal connections between these alternative extended measures, that would allow us to study them from a common formal perspective.

\section{ACKNOWLEDGEMENTS}

This work is partially supported by TIN2014-56967-R, TIN2016-77356-P, TIN2017-84804-R (Spanish Ministry of Science and Innovation) and FC-15-GRUPIN14-073 (Regional Ministry of the Principality of Asturias).

\section{REFERENCES}

[1] K. Atanassov, Intuitionistic fuzzy sets. In: VII th ITKR Session, Deposited in the Central Science and Technology Library of the Bulgarian Academy of Sciences, Sofia, Bulgaria, 1983, pp. 1684-1697.

[2] K. Atanassov, Intuitionistic fuzzy sets, Fuzzy Sets and Systems 20 (1986) 87-96.

[3] K. Atanassov, G. Gargov, Interval valued intuitionistic fuzzy sets, Fuzzy Sets and Systems 31 (1989) 343-349.

[4] K. Atanassov, Intuitionistic fuzzy sets. Theory and Applications, Springer, 1999.

[5] P. Burillo, H. Bustince, Entropy on intuitionistic fuzzy sets and on interval-valued fuzzy sets, Fuzzy Sets ad Systems 78 (1996) 305-316.

[6] H. Bustince, Indicator of inclusion grade for interval-valued fuzzy sets Application to approximate reasoning based on interval-valued fuzzy sets. International Journal of Approximate Reasoning 23 (2000) 137209.

[7] H. Bustince, E. Barrenechea, M. Pagola, Restricted equivalence functions, Fuzzy Sets ad Systems 157 (2006) 2333-2346.

[8] H. Bustince, E. Barrenechea, M. Pagola, Relationship between restricted dissimilarity functions, restricted equivalence functions and normal $E_{N^{-}}$ functions: Image thresholding invariant, Pattern Recognition Letters 29 (4) (2008) 525-536.

[9] H. Bustince, E. Barrenechea, M. Pagola, J. Fernández, Z. Xu, B. Bedregal, J. Montero, H. Hagras, F. Herrera, B. de Baets, A Historical Account of Types of Fuzzy Sets and Their Relationships, IEEE Transactions on Fuzzy Systems 24 (2016) 179-194.

[10] C. Cornelis, E. Kerre, Inclusion Measures in Intuitionistic Fuzzy Set Theory, In: T.D. Nielsen, N.L. Zhang (Eds.) Symbolic and Quantitative Approaches to Reasoning with Uncertainty, LNCS 2711, 345-356.

[11] I. Couso, H. Bustince, Three categories of set-valued generalisations from fuzzy sets to interval-valued and Atanassov intuitionistic fuzzy sets, submitted to IEEE Transactions on Fuzzy Systems, under review.

[12] I. Couso, L. Garrido, L. Sánchez, Similarity and dissimilarity measures between fuzzy sets: A formal relational study, Information Sciences 229 (2013) 122-141.

[13] I. Couso, D. Dubois, Statistical reasoning with set-valued information: Ontic vs. epistemic views, International Journal of Approximate Reasoning 55 (2014) 1502-1518.

[14] I. Couso, L. Sánchez, Additive similarity and dissimilarity measures, Fuzzy Sets and Systems 322 (2017) 35-53.

[15] L. Dengfeng, C. Chuntian, New similarity measures of intuitionistic fuzzy sets and application to pattern recognition, Pattern Recognition Letters 23 (2002) 221-225.

[16] G. Deschrijver, E.E. Kerre, On the relationship between some extensions of fuzzy set theory, Fuzzy Sets and Systems 133 (2003) 227- 235.

[17] T. Gerstenkorn and J. Mafiko, Correlation of intuitionistic fuzzy sets, Fuzzy Sets and Systems 44 (1991) 39-43.

[18] M. Galar, J. Fernández, G. Beliakov, H. Bustince, Interval-Valued Fuzzy Sets Applied to Stereo Matching of Color Images, IEEE Transactions on Image Processing 20 (2011) 1949-1961.

[19] M. B. Gorzalczany, A method of inference in approximate reasoning based on interval-valued fuzzy sets. Fuzzy sets and Systems 21(1987) $1-17$. 
[20] I. Grattan-Guinness, Fuzzy membership mapped onto interval and manyvalued quantities, Zeitschrift für mathematische Logik und Grundladen der Mathematik 22 (1976) 149-160.

[21] P. Grzegorzewski, Conditional probability and independence of intuitionistic fuzzy events, NIFS 6 (2000) 7-14.

[22] P. Grzegorzewski, Distances between intuitionistic fuzzy sets and/or interval-valued fuzzy sets based on the Hausdorff metric, Fuzzy Sets and Systems 148 (2004) 319-328.

[23] P. Grzegorzewski, On possible and necessary inclusion of intuitionistic fuzzy sets, Information Sciences 181 (2011) 342-350.

[24] W-L. Hung, M-S Yang, Similarity measures of intuitionistic fuzzy sets based on Lp metric, International Journal of Approximate Reasoning 46 (2007) 120-136.

[25] W-L. Hung, M-S. Yang, On the J-divergence of intuitionistic fuzzy sets with its application to pattern recognition, Information Sciences 178 (2008) 24 1641-1650.

[26] C-M. Hwang, M-S. Yang, W-L. Hung, M-G. Lee, A similarity measure of intuitionistic fuzzy sets based on the Sugeno integral with its application to pattern recognition, Information Sciences 189 (2012) 93109.

[27] J. Li, G. Deng, H. Li, W. Zeng, The relationship between similarity measure and entropy of intuitionistic fuzzy set, Information Sciences 188 (2012) 314-321.

[28] Z. Z. Liang and P. F. Shi, Similarity measures on intuitionistic fuzzy sets, Pattern Recognition Letters 24 (2003) 2687-2693.

[29] X.C. Liu, Entropy, distance measure and similarity measure of fuzzy sets and their relations, Fuzzy Sets and Systems 52 (1992) 305-318.

[30] A. De Luca and S. Termini, A definition of non probabilistic entropy in the settings of fuzzy set theory, Information and Control 20, (1972) 301-312.

[31] H.B. Mitchell, On the Dengfeng-Chuntian similarity measure and its appli- cation to pattern recognition, Pattern Recognition Letters 24 (2003) 3101- 3104

[32] I. Montes, N.R. Pal, V. Janis, S. Montes, Divergence measures for intuitionistic fuzzy sets, IEEE Transactions on Fuzzy Systems 23 (2015) 444-456.

[33] S. Montes, I. Couso, P. Gil, C. Bertoluzza, Divergence measure between fuzzy sets, International Journal of Approximate Reasoning 30 (2002) 91-105.

[34] A. Niewiadomski, Cylindric extensions of interval-valued fuzzy sets in data linguistic summaries, Journal of Ambient Intelligence and Humanized Computing 4 (2013) 369-376.

[35] R. Sambuc, Fonctions $\Phi$-floues. Application à l'aide au diagnostic en pathologie thyroidienne, Ph.D. Thesis, Université de Marseille, France, 1975.

[36] D. Sinha, E.R. Dougherty, Fuzzification of set inclusion: theory and applications Fuzzy Sets and Systems 55 (1993) 15-42.

[37] A. Stachowiak and K. Dyczkowski, A Similarity Measure with Uncertainty for Incompletely Known Fuzzy Sets, Fuzz IEEE 2013.

[38] E. Szmidt, J. Kacprzyk, A Concept of a Probability of an Intuitionistic Fuzzy Event, 1999 IEEE International Fuzzy Systems Conference Proceedings, 1346-1349.

[39] E. Szmidt, J. Kacprzyk, Distances between intuitionistic fuzzy sets, Fuzzy Sets and Systems 114 (2000) 505-518.

[40] E. Szmidt, J. Kacprzyk, Entropy for intuitionistic fuzzy sets, Fuzzy Sets and Systems 118 (2001) 467-477.

[41] W. Wang, X. Xin, Distance measure between intuitionistic fuzzy sets, Pattern Recognition Letters 26 (2005) 2063-2069.

[42] D. Wu, J. Mendel, Vector Similarity Measure for Interval Type-2 Fuzzy Sets, Fuzz IEEE 2007.

[43] M. Wygralak, Cardinalities of Fuzzy Sets, Springer, Berlin, Heidelberg, New York, 2003.

[44] Z.S. Xu, An overview of distance and similarity measures of intuitionistic fuzzy sets, International Journal of Uncertainty, Fuzziness and Knowledge- Based Systems 16 (2008) 529-555.

[45] Zadeh, L.A. The concept of a linguistic variable and its application to approximate reasoning-1, Information Sciences, (1975) 199-249.

[46] H. Zapata, H. Bustince, S. Montes, B. Bedregal, G.P. Dimuro, Z. Takáç, M. Baczynski, J. Fernández, Interval-valued implications and intervalvalued strong equality index with admissible orders, International Journal of Approximate Reasoning 88 (2017) 91-109

[47] W.Y. Zeng, H.X. Li, Relationship between similarity and entropy of interval valued fuzzy sets, Fuzzy Sets and Systems 157 (2006) 14771484.

[48] H. Zhang, W. Zhang, C. Mei, Entropy of interval-valued fuzzy sets based on distance and its relationship with similarity measure, KnowledgeBased Systems 22 (2009) 449-454

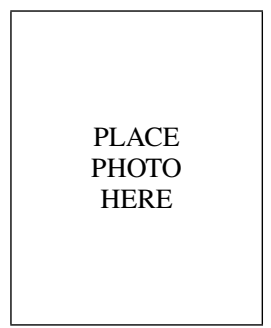

Inés Couso received the Ph.D. degree in Mathematics in 1999. Member of the Department of Statistics and O.R., University of Oviedo. She was an invited researcher at Université Paul Sabatier (Toulouse) (IRIT, 2009 and CIMI, 2015) and at Université de Montpellier 2 (LIRMM, 2011 and 2016). She currently serves as Area Editor for "Fuzzy Sets and Systems" and the "International Journal of Approximate Reasoning". Her research interests include foundations of fuzzy sets, imprecise probabilities, random sets, fuzzy random variables, statistics with coarse data and information theory.

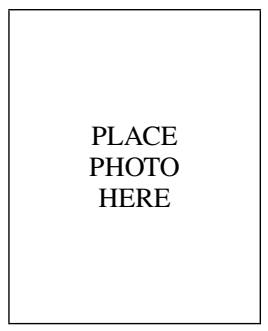

Humberto Bustince (M'08-SM'15) received the $\mathrm{Ph} . \mathrm{D}$. degree in Mathematics from the Public University of Navarra, Pamplona, Spain, in 1994. He is currently a Full Professor with the Department of Automatics and Computation of this University. $\mathrm{He}$ is the author of more than 200 published original articles. His research interests include fuzzy logic theory, extensions of fuzzy sets, fuzzy measures, aggregation functions, and fuzzy techniques for image processing. Dr. Bustince is an Editorial Board Member of IEEE Transactions on Fuzzy Systems,

Fuzzy Sets and Systems and Information Fusion. 